\title{
RESISTÊNCIA À CORROSÃO DO FILME PASSIVO FORMADO NO AÇO INOXIDÁVEL DUPLEX 2205 LAMINADO A MORNO *
}

\author{
Daniela Barçante Perasoli ${ }^{1}$ \\ Raphael França Assumpção ${ }^{1}$ \\ Davi Silva Alves ${ }^{2}$ \\ Aline Oliveira Vasconcelos Ferreira ${ }^{2}$ \\ lane Dutra Moutinho ${ }^{2}$ \\ Indiana Rosa Oliveira ${ }^{2}$ \\ Dagoberto Brandão Santos ${ }^{3}$ \\ Dalila Chaves Sicupira ${ }^{4}$
}

\section{Resumo}

O aço inoxidável duplex (AID) representa uma classe importante de aços inoxidáveis, particularmente para aplicações que exigem alta resistência mecânica e à corrosão. Sendo assim, o estudo das propriedades eletroquímicas dos filmes passivos formados em AID tem sido uma contribuição importante para a compreensão do comportamento protetor contra a corrosão. O objetivo deste trabalho é estudar o filme passivo formado no aço 2205 laminado a morno utilizando espectroscopia de impedância eletroquímica, (EIE), em ambiente de íons de cloreto. Para esse propósito, as chapas como fornecidas de AID foram laminadas a morno a $600^{\circ} \mathrm{C}$ até $60 \%$ e $80 \%$ de redução na espessura e submetidas aos testes eletroquímicos. O estudo mostra que 0 tratamento termomecânico utilizado não favoreceu a formação do filme passivo nessas condições e levou a uma menor resistência à polarização. O AID 2205 como fornecido apresentou uma maior resistência à transferência de carga na comparação com as amostras laminadas a morno.

Palavras-chave: Corrosão; Aço inoxidável; Tratamento termomecânico; EIE.

\section{CORROSION RESISTANCE OF PASSIVE FILM FORMED ON WARM ROLLED 2205 DUPLEX STAINLESS STEEL}

\begin{abstract}
Duplex stainless steel (DSS) represent an important class of stainless steels particularly for applications requiring high mechanical and corrosion resistance. Therefore, the study of electrochemical properties of passive films formed in DSS has been an important contribution to understand the protective behavior against corrosion. The objective of this work is to study the passive film formed in the warm rolled 2205 DSS using electrochemical impedance spectroscopy (EIS) in the chlorine ion environment. For this purpose, the duplex stainless steel were warm rolled at $600^{\circ} \mathrm{C}$ to $60 \%$ and $80 \%$ reduction in thickness and submitted to electrochemical tests. The study shows that the thermomechanical treatment used did not favor the formation of the passive film under these conditions and led to a lower polarization resistance. The 2205 DSS as received exhibited a higher charge transfer resistance compared to the warm rolled samples.
\end{abstract}

Keywords: Corrosion; Stainless steel; Thermomechanical treatment; Electrochemical Impedance.

1 Engenharia Metalúrgica, Graduando, Estudante, Departamento de Engenharia Metalúrgica e Materiais, Universidade Federal de Ouro Preto, Ouro Preto, MG, Brasil.

2 Engenharia Metalúrgica, Graduando, Estudante, Departamento de Engenharia Metalúrgica e Materiais, Universidade Federal de Minas Gerais, Belo Horizonte, MG, Brasil.

3 Engenharia Metalúrgica, Doutor, professor, Departamento de Engenharia Metalúrgica e de Materiais, Universidade Federal de Minas Gerais, Belo Horizonte, MG, Brasil.

4 Química, Doutora, Professora, Departamento de Química, Universidade Federal de Ouro Preto, Ouro Preto, MG, Brasil. 


\section{INTRODUÇÃO}

Os aços inoxidáveis duplex (AID) estão sendo amplamente utilizados como alternativas para aços inoxidáveis austeníticos e ligas à base de níquel [1]. Rrepresentam uma classe importante de aços com uma combinação de resistência mecânica e à corrosão em ambientes contendo íons cloreto [2]. Os AID apresentam uma microestrutura formada por quantidades aproximadamente iguais de austenita $(\gamma)$ e ferrita ( $\alpha$ ) e suas melhores propriedades permitem que sejam muito utilizados na indústria química, petroquímica, marítima, nuclear e de papel $[3,4]$.

Os elementos de ligas são os responsáveis pelo equilíbrio entre as fases. Os principais elementos de liga são carbono, cromo, níquel, molibdênio, tungstênio, nitrogênio e cobre, não são distribuídos uniformemente na ferrita e austenita. Cromo, molibdênio e tungstênio enriquecem na ferrita, enquanto carbono, níquel, nitrogênio e cobre são concentrados na austenita [5].

A alta resistência à corrosão é devida à formação espontânea de uma camada de óxido de cromo sobre a superfície de aço. Embora extremamente fina, esta película invisível é firmemente aderida ao metal e é extremamente protetora em uma ampla gama de ambientes corrosivos. Esta película é rapidamente restaurada, na presença de oxigênio, e, assim, danos de abrasão ou cortes são reparados rapidamente [6].

Estudos recentes confirmaram que o filme de óxido passivo formado em aços inoxidáveis consiste principalmente de óxido de cromo, $\mathrm{Cr}_{2} \mathrm{O}_{3}$ e óxido de ferro, $\mathrm{Fe}_{2} \mathrm{O}_{3}$, como camadas interna e externa, respectivamente $[7,8]$. O primeiro atua como uma camada de barreira contra a transferência de cátions e o último é uma camada de troca com o eletrólito. Geralmente, os filmes passivos dos metais são compostos principalmente de óxidos ou hidróxidos metálicos, que são semicondutores $[7,8]$.

A deterioração da resistência à corrosão resultante de tratamentos termomecânico, como a laminação a morno, é um problema típico para os utilizadores de AID [9]. O propósito do processo de laminação é reduzir a espessura da peça de trabalho da espessura inicial para uma espessura final pré-determinada. A medida que a laminação progride, as propriedades mecânicas do material mudam acentuadamente [9].

O conhecimento do comportamento eletroquímico desses materiais é imprescindível para a sua aplicação industrial, uma vez que os materiais são constantemente submetidos a ambientes e condições agressivas de serviço e a vida útil de muitos tipos de equipamentos depende da detecção precoce de problemas. A evolução da pesquisa sobre as propriedades eletroquímicas dos filmes passivos formados em aços inoxidáveis tem sido uma contribuição importante para a compreensão do comportamento protetor contra a corrosão dessas ligas. Nesse contexto, o objetivo deste trabalho é estudar o filme passivo formado no aço UNS S32205 laminado a morno utilizando a técnica de espectroscopia de impedância eletroquímica (EIE).

\section{MATERIAIS E MÉTODOS}

O aço inoxidável duplex UNS S32205 foi fornecido pela empresa Aperam South America na forma de chapas laminadas a quente com $5,1 \mathrm{~mm}$ de espessura, 150 $\mathrm{mm}$ de comprimento e $100 \mathrm{~mm}$ de largura. As chapas do material como fornecidas foram laminadas a morno a $600^{\circ} \mathrm{C}$ com redução de 60 e $80 \%$ de espessura. A composição química do aço se encontra na Tabela 1 abaixo. 
Tabela 1. Composição química (\% em massa do aço inoxidável UNS S32205

\begin{tabular}{cccccccccc} 
Elemento & $\mathbf{C}$ & $\mathbf{M n}$ & $\mathbf{S i}$ & $\mathbf{C r}$ & $\mathbf{N i}$ & $\mathbf{M o}$ & $\mathbf{N}$ & $\mathbf{P}$ & $\mathbf{S}$ \\
\hline \% em massa & 0,026 & 1,83 & 0,25 & 22,43 & 5,44 & 3,04 & 0,15 & 0,03 & 0,0002 \\
\hline
\end{tabular}

Os testes de espectroscopia de impedância eletroquímica (EIE) foram realizados em uma célula convencional de três eletrodos, sendo utilizado um eletrodo de referência de $\mathrm{Ag} / \mathrm{AgCl}$ e um contra eletrodo de platina, em solução de $\mathrm{NaCl} 3,5 \%$ à temperatura ambiente. Para a confecção do eletrodo de trabalho, chapas de AID foram cortadas em amostras de dimensões $10 \times 10 \mathrm{~mm}$ e embutidas a frio em resina acrílica. $O$ contato elétrico foi feito soldando um fio metálico na superfície da amostra. Os testes foram realizados segundo a Norma ASTM G 106 [10].

Antes de cada experimento, os eletrodos de trabalho foram lixados utilizando as lixas de 100, 240, 320, 400, 600 mesh, desengordurados com detergente, álcool e lavados em água. A região de contato entre a amostra e a resina foi selada e as amostras foram armazenadas no dessecador.

Os experimentos foram feitos utilizando um potenciostato PGSTAT302N, acoplado a um analisador de resposta de frequência FRA32M da marca AUTOLAB.

Para os testes de EIE usou-se uma amplitude de perturbação no potencial de $10 \mathrm{mV}$ e 10 pontos por década de frequência no intervalo de $100 \mathrm{kHz}$ a $5 \mathrm{mHz}$. As medidas foram iniciadas após $1 \mathrm{~h}$ de imersão para estabilização do potencial de circuito aberto (OCP). Os dados de impedância foram analisados utilizando circuitos elétricos equivalentes e ajustados usando o programa ZView (Scribner Assoc.). Os testes foram feitos em triplicata.

\section{RESULTADOS E DISCUSSÃO}

Os resultados de impedância eletroquímica fornecem informações relacionadas ao processo eletroquímico que ocorre no sistema em estudo. A interpretação dos dados de impedância é feita através da análise de circuitos elétricos equivalentes. Neste trabalho utilizou-se o circuito equivalente constituído pelo arranjo ([Rs $\left.\left(C P E|| R_{c t}\right)\right]$ ) (Figura 1), em que o elemento $R_{s}$ é a resistência da solução, CPE é o elemento de fase constante e $R_{c t}$ é a resistência à transferência de carga na interface metal/eletrólito.

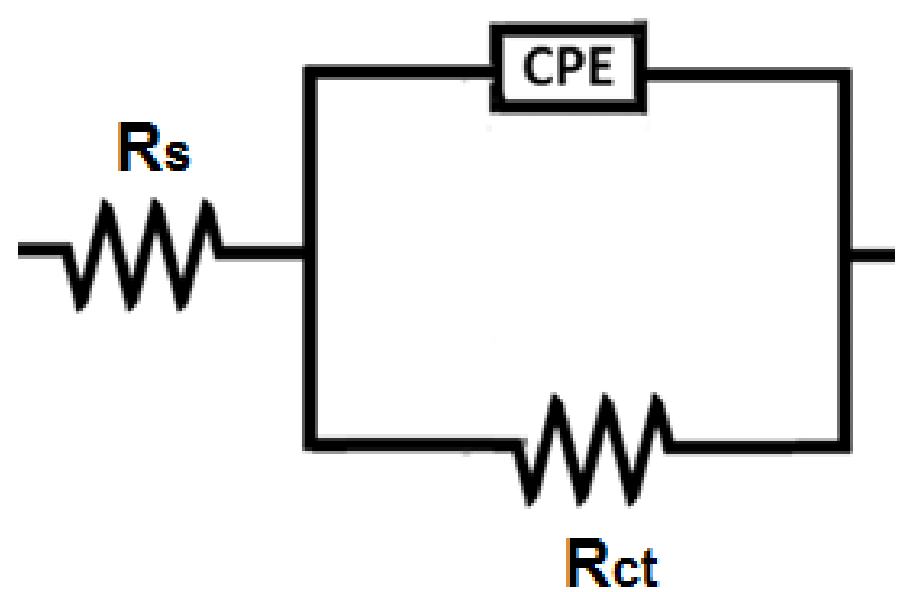

Figura 1. Circuito equivalente usado para modelagem dos dados de impedância. 
A Figura 2(a-c) mostra o diagrama de Nyquist (a) e Bode (b e c) para as amostras laminadas a morno e para o aço como fornecido.
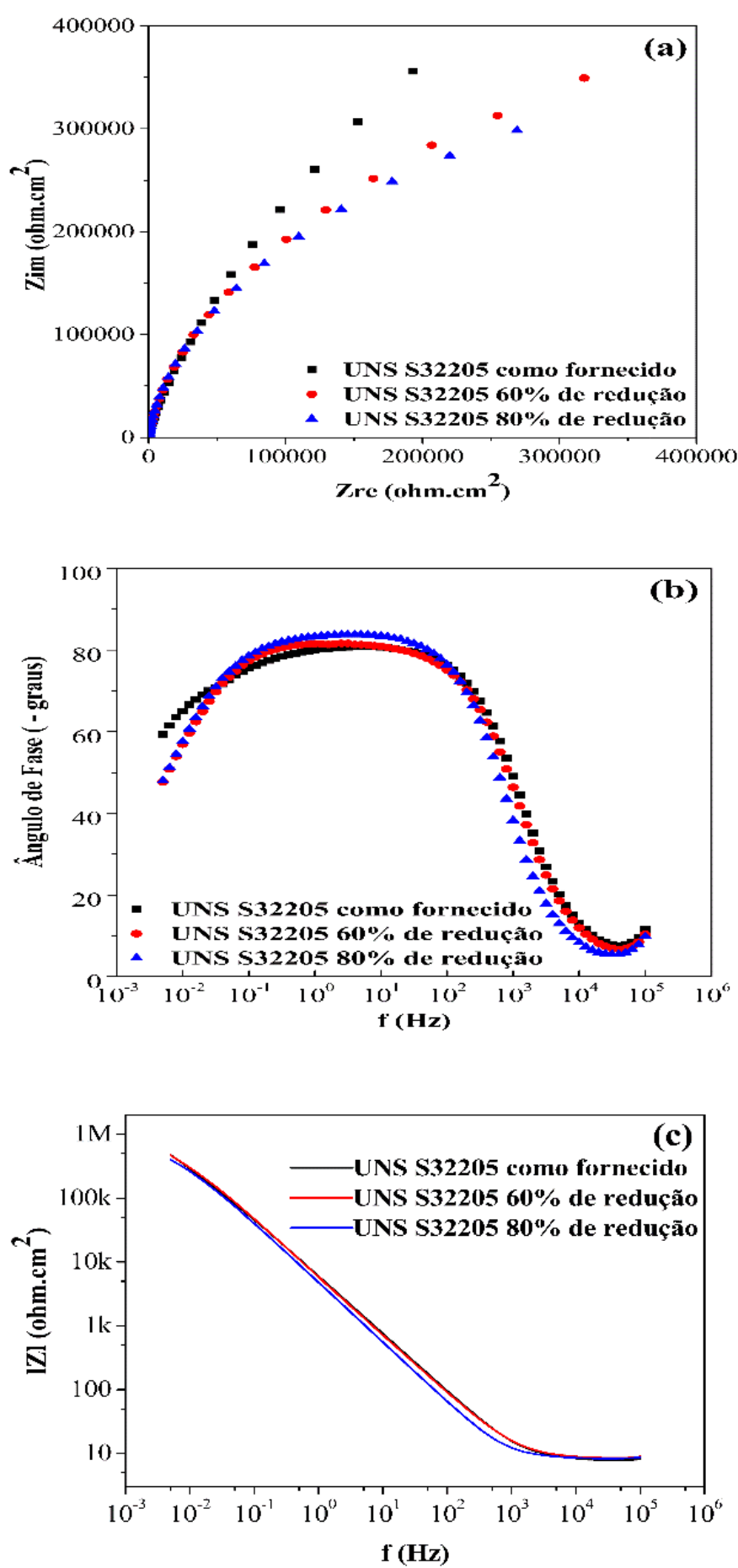

Figura 2. Resultados de EIE: a) Diagrama de Nyquist e Diagrama de Bode: b) Ângulo de fase c) Módulo de Z.

O diagrama de Nyquist (Fig. 2a) mostrou uma diferença no diâmetro do semicírculo obtido para o aço CF e as amostras laminadas a morno, que indica um processo corrosivo mais lento para o aço CF. A Fig. 2b mostra altos valores de ângulo de fase ao longo de uma larga faixa de frequência. Segundo Brytan, Niagaj and Reiman [11] isso é um comportamento típico dos aços inoxidáveis que são materiais com 
capacidade de passivação devido à formação de uma camada passiva na superfície dos mesmos.

Como pode ser observado na Figura 2b, o diagrama de Bode apresenta apenas uma constante de tempo (somente um máximo no ângulo de fase na região de frequência média). Além disso, os valores de ângulo de fase próximos de -80 graus indicam a formação e o crescimento de uma camada passiva. Comportamento similar foi obtido por outros autores em aços inoxidáveis ferríticos em solução $0,1 \mathrm{~mol} / \mathrm{L} \mathrm{HNO}_{3}$ [12].

Os elementos que compõem o circuito elétrico correspondem às propriedades eletroquímicas do sistema corrosivo, incluindo a resistência à transferência de carga. Para obter essas propriedades, os dados experimentais foram ajustados utilizandose o programa Zview e os parâmetros resultantes estão apresentados na Tabela 2 abaixo.

Tabela 2. Parâmetros do ajuste dos dados de EIE para o aço 2205 como fornecido e laminado a morno.

\begin{tabular}{lcccccc}
\hline & \multicolumn{2}{c}{$\mathbf{C F}$} & \multicolumn{2}{c}{$\mathbf{6 0 \%}$} & \multicolumn{2}{c}{$\mathbf{8 0 \%}$} \\
\cline { 2 - 7 } & Valor & Desvio & Valor & Desvio & Valor & Desvio \\
\hline $\mathbf{R}_{\mathbf{s}}\left(\boldsymbol{\Omega} \cdot \mathbf{c m}^{2}\right)$ & 8,204 & 0,811 & 8,557 & 0,480 & 7,072 & 1,128 \\
\hline $\mathbf{R}_{\mathbf{c t}}\left(\mathbf{k} \boldsymbol{\Omega} \cdot \mathbf{c m}^{2}\right)$ & 1100,0 & 55,6 & 734,0 & 97,6 & 577,0 & 162,0 \\
\hline $\mathbf{Q}\left(\boldsymbol{\mu} \boldsymbol{\Omega}^{-1} \cdot \mathbf{c m}^{-\mathbf{2}} \cdot \mathbf{s}^{\mathbf{n}}\right)$ & 34,700 & 0,554 & 33,90 & 0,85 & 40,10 & 0,83 \\
\hline $\mathbf{n}$ & 0,8885 & 0,0028 & 0,8989 & 0,0288 & 0,9183 & 0,0105 \\
\hline
\end{tabular}

A modelagem de dados de impedância mais complexos, como no caso de semicírculos imperfeitos, requer o uso de circuitos com elemento de fase constante (CPE) para representar a dispersão da capacitância e este é caracterizado pelos parâmetros $n$ e $Q \mathrm{O}$ parâmetro $\mathrm{n}$ descreve o desvio do comportamento capacitivo ideal e $Q\left(\mu \mathrm{F} . \mathrm{cm}^{-2} \cdot \mathrm{s}^{\mathrm{n}}\right)$ é equivalente à capacitância do filme quando o valor de $\mathrm{n}$ se aproxima da unidade e o comportamento de um capacitor ideal é observado [13].

A resistência à transferência de carga foi maior para o aço 2205 CF quando comparado às amostras laminadas a morno (Tabela 2). Isso sugere que 0 tratamento termomecânico utilizado prejudicou a resistência à corrosão do filme passivo. Com relação às amostras laminadas, a resistência à corrosão diminuiu com o aumento da deformação.

De acordo Fattah-alhosseini e Vafaeian [12] a alteração do valor de $Q$ para o filme passivo é um sinal de mudança na espessura do filme passivo formado, ou seja, há uma relação entre a espessura do filme passivo e o valor do parâmetro $Q$. Como pode ser observado na Tabela 2, o valor de $Q$ aumenta para uma maior redução de espessura na laminação, indicando que o tratamento termomecânico utilizado não favoreceu a formação do filme passivo nessas condições.

\section{CONCLUSÔES}

A resistência à transferência de carga na interface metal/eletrólito $\left(R_{c t}\right)$ foi maior para o aço inoxidável duplex 2205 como fornecido quando comparado às amostras laminadas a morno. Dentre as amostras laminadas a morno, o aumento da deformação de $60 \%$ para $80 \%$ de redução de espessura resultou em menores valores de $R_{\text {ct }}$ para o aço UNS S32205.

Os resultados obtidos nos testes de espectroscopia de impedância eletroquímica (EIE) mostraram que o tratamento termomecânico levou a uma menor resistência à 
polarização. O tratamento termomecânico utilizado não favoreceu a formação do filme passivo nessas condições.

\section{Agradecimentos}

Os autores agradecem a Aperam South America pelo fornecimento do aço de estudo. Também agradecem a CAPES-PROEX-PPGEM/UFMG, ao CNPq e a FAPEMIG pela concessão de bolsas de IC e auxílio financeiro.

\section{REFERÊNCIAS}

1 Jinlong L, Tongxiang L, Chen W, Limin D. Effect of ultrafine grain on tensile behaviour and corrosion resistance of the duplex stainless steel. Materials Science and Engineering C. 2016;62:558-563.

2 Sicupira DC, Cardoso Junior R, Bracarense AQ, Frankel GS, Lins VFC. Cyclic Polarization Study of Thick Welded Joints of Lean Duplex Stainless Steel for Application in Biodiesel Industry. Materials Research. 2017;20(1):161-167.

3 Sicupira DC, Cardoso Junior R, Bracarense AQ, Frankel GS, Lins VFC. Electrochemical study of passive films formed on welded lean duplex stainless steel. Materials and Corrosion. 2016;68(6):604-612.

4 Ahmed MZ, Bhattacharjee PP. Microstructure, Texture, and Tensile Properties of a Severely Warm-Rolled and Annealed Duplex Stainless Steel. Metallurgical and Materials Transactions A. 2016;87(4):472-483.

5 Chen L, Tan $\mathrm{H}$, Wang Z, Li J, Jiang Y. Influence of cooling rate on microstructure evolution and pitting corrosion resistance in the simulated heat-affected zone of 2304 duplex stainless steels. Corrosion Science. 2012;58:168-174.

6 Ibarra M, Núñez E, Huerta J. Manual Aceros Inoxidables. Cerrillos: 2M Impresores Ltda; 2010.

7 N. E. Hakiki, Comparative study of structural and semiconducting properties of passive films and thermally grown oxides on AISI 304 stainless steel. Corrosion Science. 2011; 53(9):2688-2699.

8 Zheng ZJ, Gao Y, Gui Y, Zhu M. Studying the fine microstructure of the passive film on nanocrystalline 304 stainless steel by EIS, XPS, and AFM. Journal of Solid State Electrochemistry. 2014;18(8):2201-2210.

9 Lenard JG. Primer on Flat Rolling. 2nd ed. Waterloo: Elsevier; 2014.

10 ASTM G106-86. Standard practice for Verification of Algorithm and Equipment for Electrochemical Impedance Measurements. West Conshohocken: ASTM International; 1999.

11 Brytan Z, Niagaj J, Reiman L. Corrosion studies using potentiodynamic and EIS electrochemical techniques of welded lean duplex stainless steel UNS S82441. Applied Surface Science. 2016;388(A):160-168.

12 Fattah-alhosseini A. Vafaeian S. Comparison of electrochemical behavior between coarse-grained and fine-grained AISI 430 ferritic stainless steel by Mott-Schottky analysis and EIS measurements. Journal of Alloys and Compounds. 2015;639:301-307.

13 BenSalah M, Sabot R, Triki E, Dhouibi L. Refait PH, Jeannin M. Passivity of Sanicro28 (UNS N-08028) stainless steel in polluted phosphoric acid at different temperatures studied by electrochemical impedance spectroscopy and Mott-Schottky analysis. Corrosion Science. 2014;86:61-70. 